\title{
Kurze KVT bei Dermatillomanie ausreichend
}

Bisher ist die Dermatillomanie wenig erforscht. Die Behandlung ist ähnlich zu Behandlungen anderer Impulskontrollstörungen wie der Trichotillomanie. Empfohlen wird eine kognitive Verhaltenstherapie. Die Ergebnisse der folgenden Studie deuten darauf hin, dass eine umfangreiche Behandlung der Dermatillomanie nicht erforderlich ist, sondern bereits vier Sitzungen kognitive Verhaltenstherapie eine bleibende Verbesserung erzielen.

$\mathrm{n}$ der Studie von Schuck et al. wurden 34 Universitätsstudenten, die unter Dermatillomanie litten, zufällig einer Behandlungs- oder Wartelistengruppe (je $\mathrm{n}=17)$ zugewiesen. In der Behandlungsgruppe erhielten die Betroffenen je vier Sitzungen standardisierte und manualisierte kognitive Verhaltenstherapie (Psychoedukation, Identifikation dysfunktionaler Kognitionen bezogen auf die Dermatillomanie, Steigerung der Selbstkontrolle und Rückfallprophylaxe).

Untersucht wurde eine große Bandbreite von Outcome-Variablen, wie die
Schwere der Hautmanipulation, deren psychosoziale Auswirkungen sowie die Stärke dysfunktionaler Kognitionen. Die Outcome-Variablen wurden vor, unmittelbar nach sowie zwei Monate nach der Behandlung erhoben.

\section{Konsistente Effekte mit relativ} wenigen KVT-Sitzungen erreichbar Die Teilnehmer der Behandlungsbedingung zeigten einen im Vergleich zur Wartebedingung signifikant stärkeren Rückgang aller gemessenen Variablen. Für alle Ergebnismaße wurden große Effektstärken zwischen $\mathrm{d}=0,90$ und $\mathrm{d}=1,89$ erzielt. Patienten berichteten, vermehrt dem Impuls zu kratzen etc. widerstehen zu können. Diese Behandlungseffekte konnten über einen Zeitraum von zwei Monaten aufrechterhalten werden. Der Einsatz zusätzlicher kognitiver Interventionen zur klassischen Verhaltenstherapie hat vermutlich zu den hervorragenden Ergebnissen beigetragen.

Kommentar: Hervorzuheben ist, dass mit relativ wenigen Sitzungen KVT konsistente Effekte erzielt werden konnten. Die Ergebnisse deuten darauf hin, dass eine umfangreiche Behandlung der Dermatillomanie nicht erforderlich ist.

Dipl.-Psych. Nicola Stelzer

Schuck K et al. The effects of brief cognitivebehaviour therapy for pathological skin picking: A randomized comparison to wait-list control. Behaviour Research and Therapy 2011; 49: 11e17

\section{Zwanghafte Persönlichkeitsstörung erschwert die Behandlung der Zwangsstörung}

Bis zu einem Drittel der Patienten mit Zwangsstörungen weist auch die Merkmale der zwanghaften (anankastischen) Persönlichkeitsstörung auf. Die vorliegende Studie zeigt erstmals, dass das ein Prädiktor für ein schlechteres Therapieergebnis einer Expositionsbehandlung mit Reaktionsmanagement ist.

u der Frage, ob eine komorbide zwanghafte Persönlichkeitsstörung (OCPD) auch das Behandlungsergebnis bei Zwangsstörungen verschlechtert, exis-tierten bislang nur einige wenige Arbeiten mit zudem widersprüchlichen Ergebnissen. Komorbiditätsstudien haben in der Vergangenheit überzeugend auf einen Zusammenhang zwischen OCPD und Zwangsstörungen (OCD) hingewiesen. So hätten nach DSM-IV Kriterien 23-32\% aller OCD-Patienten eine komorbide OCPD, umgekehrt litten $21 \%$ der OCPD-Patienten auch unter einer Zwangsstörung.

In der vorliegenden Arbeit wollten die Autoren erstmals spezifisch den Einfluss einer OCPD auf den Erfolg einer Expositionsbehandlung mit Reaktionsmanagement bei Zwangsstörungen untersuchen.

\section{Vorhergehende Studien zum Thema} zwanghafte Persönlichkeitsstörung

Für psychische Störungen der Achse I wie chronische Depressionen, Anorexia Nervosa und die generalisierte Angststörung, wurde bereits gezeigt, dass deren Prognose durch eine komorbide OCPD ungünstig beeinflusst wird.

Die Studie von Baer et al. [1] zeigte, dass zwar schizotypische, Borderlineund vermeidende Persönlichkeitsstörungen, nicht aber eine OCPD, das Ansprechen auf Clomipramin verschlechterten. Die Studie von Cavedini et al. [2] demonstrierte einen solchen ungünstigen Einfluss auf eine Behandlung mit SRI dagegen gerade für die OCPD. Dass die psychotherapeutische Behandlung der OCD mittels individuell zugeschnittener, multimodaler kognitiver Verhaltenstherapie nur durch schizotypische und passiv-aggressive Persönlichkeitszüge ungünstig beeinflusst wird, wies die Studie von Fricke et al. nach [3].

\section{Teilnehmer hatten eine mindes- tens ein Jahr andauernde OCD}

Für die vorliegende Studie werteten die Wissenschaftler die Daten einer früheren Arbeit von Simpson et al. [4] erneut aus, die die Augmentation einer Psychopharmakotherapie durch störungsspezifische Psychotherapie untersucht hatte. Diesmal analysierten sie aber nur die Daten der Interventionsgruppenteilnehmer $(\mathrm{n}=54)$, die seit mindestens einem Jahr an einer OCD erkrankt waren $(n=49)$. Es handelte sich um erwachsene, ambulante Patienten, die seit mindestens zwölf Wochen stabil auf einen SRI eingestellt waren (therapeutische Dosierung), sich darunter wenigstens minimal verbessert hatten, jedoch weiterhin mindestens moderate Zwänge zeigten. Die Patienten hatten (unter weiterer Einnahme des SRI) nach zwei einführenden Sitzungen über acht Wochen insgesamt 15 jeweils eineinhalb- bis zweistündige Sitzungen mit Expositionsbehandlung erhalten.

Um den Schweregrad der Zwangsstörung zu überprüfen, wurde die Yale- 